Check for updates

Cite this: RSC Adv., 2017, 7, 30657

Received 18th February 2017

Accepted 21st May 2017

DOI: $10.1039 / c 7 r a 02049 j$

rsc.li/rsc-advances

\title{
A novel organosulfur compound, thiacremonone, regulates RANKL-induced osteoclast differentiation
}

\author{
Kyung-Ran Park, ${ }^{a}$ Ji-Youn Kim, ${ }^{b}$ Jin Tae Hong ${ }^{c}$ and Hyung-Mun Yun (D)*d
}

\begin{abstract}
The medicinal properties of functionally active organosulfur compounds have received great attention for applications in treating various diseases. Thiacremonone (2,4-dihydroxy-2,5-dimethyl-thiophene-3-one, THIA) is a novel organosulfur compound generated from high-temperature-high-pressure-treated garlic and has been reported to display pharmacological effects against various diseases. However, the role of THIA in bone resorbing cells was not reported. Herein, we have examined the pharmacological properties of THIA on osteoclast differentiation in primary cultured bone marrow macrophages (BMMs). Our results showed that THIA suppressed receptor activator of NF- $\kappa B$ ligand (RANKL)-induced gene expression (c-Fos and NF-ATC1) for osteoclast differentiation under no cytotoxicity condition. In addition, THIA significantly inhibited TRAP positive multinucleated osteoclasts and F-actin ring formation during RANKL-mediated osteoclastogenesis. However, in primary cultured calvarial pre-osteoblasts, THIA did not influence the cytotoxicity and osteoblast differentiation, as assessed from the alkaline phosphatase (ALP) activity and mineralized nodule formation. Our findings have suggested that THIA can be considered as a potential agent for the therapeutics of bone diseases such as osteoporosis, alveolar bone resorption, and osteoarthritis.
\end{abstract}

\section{Introduction}

Bone metabolism is a physiological process that maintains the skeleton by removing and replacing old bones. ${ }^{1}$ An impaired osteoclast differentiation and function may cause osteoporosis, which is one of the most common bone diseases that can be characterized by decreased bone mass, increased bone fragility, and increased risk of fractures. ${ }^{1-3}$ Bisphosphonates, receptor activator of $\mathrm{NF}-\kappa \mathrm{B}$ ligand (RANKL) inhibitor, and antiresorptives have been used in the main therapies based on increasing the bone mass in bone-destructive diseases. ${ }^{4}$ However, their ability to repair or recover bone mass has only modest effects as well as the drugs are comparatively expensive, difficult to administer and have adverse effects. ${ }^{5,6}$ Therefore, it would be helpful to develop novel and effective anabolic agents that can directly inhibit osteoclast-mediated bone resorption.

Organosulfur compounds are known to possess medicinal properties and health benefits of garlic as they exhibit biological

${ }^{a}$ Department of Oral \& Maxillofacial Regeneration, Graduate School, Kyung Hee University, Seoul 130-701, Republic of Korea

${ }^{b}$ Department of Dental Hygiene, College of Health Science, Gachon University, Incheon, 406-799, Republic of Korea

${ }^{c}$ College of Pharmacy and Medical Research Center, Chungbuk National University, Chungbuk 361-763, Republic of Korea

${ }^{d}$ Department of Oral and Maxillofacial Pathology, School of Dentistry, Kyung Hee University, 1 Heogi-Dong, Dongdaemun-Gu, Seoul 130-701, Republic of Korea. E-mail: yunhm@khu.ac.kr; Fax: +82-02-960-1457; Tel: +82-02-961-0343 activities such as anti-cancer, anti-neurological disease and anti-osteoarthritis activities. ${ }^{7}$ Among them, we have been focusing on thiacremonone (2,4-dihydroxy-2,5-dimethylthiophene-3-one, THIA) isolated from heated garlic. Our studies have also demonstrated the effects of THIA as promising candidates for drugs in the treatment of cancer, arthritic diseases, neurological diseases, and liver diseases. ${ }^{8-11}$ However, the potential effects of THIA on bone remodeling and bone diseases have not yet been established.

Therefore, in the present study, we have investigated the pharmacological properties of THIA on in vitro cell system using primary mouse bone marrow macrophages (BMMs) and calvarial pre-osteoblasts.

\section{Materials and methods}

\section{Extraction and characterization of thiacremonone}

The structure of a sulfur compound isolated from garlic (thiacremonone) has been shown in Fig. 1A. Garlic (Allium sativum L.) was heated at a temperature of $130{ }^{\circ} \mathrm{C}$ for $2 \mathrm{~h}$. The heated samples were ground to a juicy form and then filtered on a Buchner funnel under a vacuum. Heated garlic juice was separated consecutively in a separating funnel using ethyl acetate. Isolated compounds from the ethyl acetate layer of the heated garlic juice were subjected to column chromatography on silica gel. This fraction containing thiacremonone was 
A

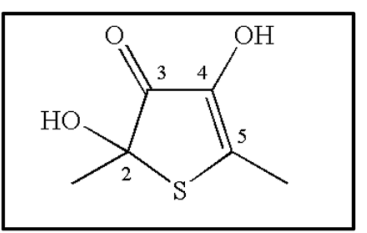

B
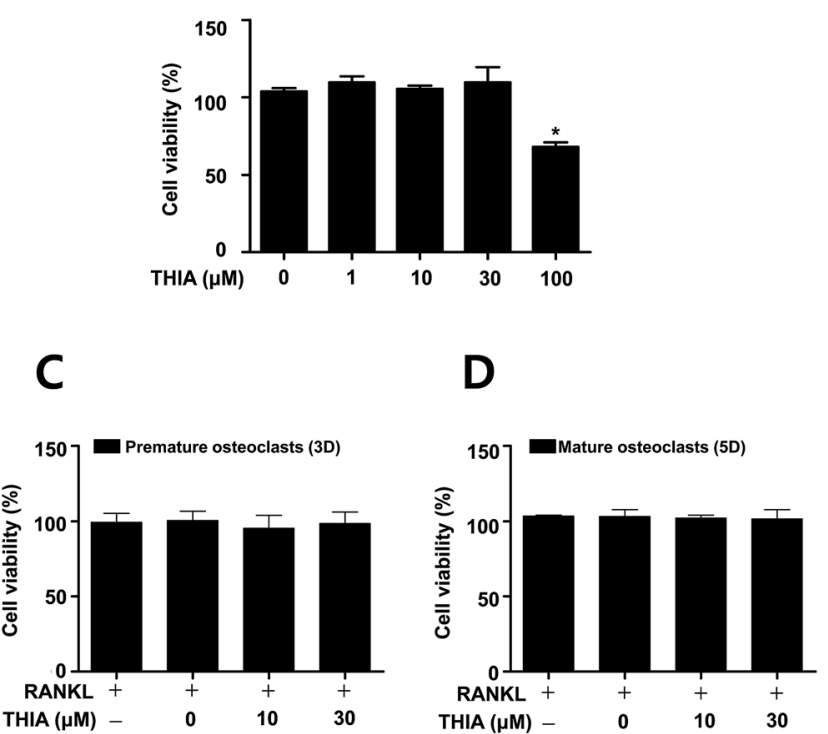

Fig. 1 Effects of THIA on cytotoxicity in BMMs and osteoclasts. (A) Chemical structures of THIA, thiacremonone. (B) After primary mouse bone marrow macrophages (BMMs) were seeded onto 96 -well plates, THIA was treated in the cells with 1, 10, 30, and $100 \mu \mathrm{M}$ for $24 \mathrm{~h}$. (C and D) The BMMs were cultured with THIA in $100 \mathrm{ng} \mathrm{mL}^{-1}$ RANKL for 3 days (C) and 5 days (D). Cell viability was measured using the MTT assay. $*, p<0.05$ compared to control. Data are representative of three independent experiments

purified by preparative RP-HPLC on a Younglin SP930D Instrument. $^{12}$

\section{Live subject statement}

All the mice used in the present study were maintained in accordance to the National Institute of Toxicological Research of the Korea Food and Drug Administration guidelines for the humane care and use of laboratory animals. All experimental procedures in the current study were approved by Kyung Hee University Animal Care Committee (approval number: KHMCIACUC 2015-002).

Primary culture of bone marrow macrophages, and osteoclast differentiation

Mouse bone marrow cells isolated by flushing the marrow space of femur and tibia from 5 weeks old mice were incubated overnight on culture dishes in $\alpha$-MEM (Gibco Laboratories) containing $10 \%$ FBS (Gibco Laboratories) and antibiotics (100 units per $\mathrm{mL}$ penicillin $\mathrm{G}$ and $100 \mu \mathrm{g} \mathrm{mL}{ }^{-1}$ streptomycin) at $37{ }^{\circ} \mathrm{C}$ in a humidified atmosphere of $5 \% \mathrm{CO}_{2}$ and $95 \%$ air. After discarding the adherent cells, the floating cells were further incubated with mouse M-CSF (30 ng $\mathrm{mL}^{-1}$ ) on Petri dishes. BMMs became adherent after a 3 days culture and then the cells were differentiated into osteoclasts using mouse RANKL (100 ng $\mathrm{mL}^{-1}$ ) and M-CSF (30 ng $\mathrm{mL}^{-1}$ ) for 5 days.

\section{Primary culture of mouse calvarial pre-osteoblasts, and} osteoblast differentiation

Primary osteoblasts were isolated from the calvariae of 1 day-old ICR mice after dissecting aseptically and treated with $0.2 \%$ collagenase-dispase enzyme solution (Sigma-Aldrich, St. Louis, MO). Cells (passage 0) were collected by centrifugation after repeated digestions six times and cultured in $\alpha$-minimum essential medium ( $\alpha$-MEM) (Gibco Laboratories, Grand Island, $\mathrm{NY}$ ) without L-ascorbic acid supplemented with $10 \%$ fetal bovine serum (FBS), penicillin (100 units per $\mathrm{mL}$ ), and streptomycin $\left(100 \mu \mathrm{g} \mathrm{mL}^{-1}\right)$ at $37{ }^{\circ} \mathrm{C}$ in a humidified atmosphere of $5 \% \mathrm{CO}_{2}$ and $95 \%$ air. The cells were detached and reseeded at approximately $70-80 \%$ confluence, and then the cells (passage 1) were used for the experiments. ${ }^{13}$ Osteoblast differentiation was induced by changing the osteogenic supplement medium (OS) containing $50 \mu \mathrm{g} \mathrm{mL}{ }^{-1}$ L-ascorbic acid and $10 \mathrm{mM} \beta$-glycerophosphate when the cells were approximately $90 \%$ confluent. The medium was replaced every 2 days during the incubation period.

\section{MTT assay}

Cell toxicity was measured by 3-[4,5-dimethylthiazol-2-yl]-2,5diphenyltetrazolium bromide (MTT) assay to detect NADHdependent dehydrogenase activity. ${ }^{\mathbf{1 4}}$ Fifty microliters of MTT solution ( $5 \mathrm{mg} \mathrm{mL}^{-1}$ ) in $1 \mathrm{X}$ phosphate-buffered saline (PBS) was directly added to the cells, which was then incubated for $2 \mathrm{~h}$ to allow MTT to metabolize to formazan. Absorbance was measured at a wavelength of $540 \mathrm{~nm}$ using an enzyme linked immunosorbent assay (ELISA) reader (Beckman Coulter, Fullerton, CA).

\section{Tartrate-resistant acid phosphatase (TRAP) staining}

After BMMs were differentiated into osteoclasts for 5 days, the cells were fixed with $4 \%$ formaldehyde for $15 \mathrm{~min}$. The cells were washed and stained for TRAP using a leukocyte acid phosphatase cytochemistry kit (Sigma) as per the manufacturer's instructions. The TRAP-positive multinucleated cells (MNCs) containing three or more nuclei were counted as mature osteoclasts using a light microscope. Typically, cells were mostly TRAP-positive and mononuclear at 2 days after induction with RANKL and TRAP-positive and multinuclear 2 or 3 d later. We considered mononuclear TRAP-positive cells to be preosteoclasts and multinuclear TRAP-positive cells to be mature osteoclasts as described earlier. ${ }^{15}$

\section{F-Actin-ring formation analysis}

Osteoclasts were fixed with $4 \%$ formaldehyde, permeabilized with $0.1 \%$ Triton $\mathrm{X}-100$, and incubated with FITC-phalloidin (Invitrogen) for $30 \mathrm{~min}$. After being washed with PBS, the cells 
were incubated with $1 \mu \mathrm{g} \mathrm{mL}^{-1}$ DAPI (Sigma-Aldrich) and washed three times and viewed on a fluorescence microscope (Carl Zeiss, Oberkochen, Germany).

\section{Alkaline phosphatase (ALP) activity}

ALP activity was measured via spectrophotometry. Cells were homogenized in $0.5 \mathrm{~mL}$ distilled water using a sonicator and centrifuged. The aliquots of cell homogenate were incubated with $15 \mathrm{~mm} p$-NPP in $0.1 \mathrm{M}$ glycine-NaOH (pH 10.3) at $37^{\circ} \mathrm{C}$ for $30 \mathrm{~min}$. The reaction was stopped by adding $0.25 \mathrm{~N} \mathrm{NaOH}$. The absorbance was measured at $405 \mathrm{~nm}$ using an ELISA reader (Beckman Coulter).

\section{Alizarin red S (ARS) staining}

After 14 days of culture, cells were fixed in 70\% ice-cold ethanol for $1 \mathrm{~h}$ and rinsed with distilled water. Cells were stained with $40 \mathrm{mM}$ Alizarin red $\mathrm{S}(\mathrm{pH} 4.2)$ for $10 \mathrm{~min}$ with gentle agitation. The level of Alizarin red s staining was observed under light microscopy. Stains were eluted with $100 \%$ DMSO to quantify the amount of alizarin red staining and measured at $590 \mathrm{~nm}$.

\section{Quantitative real-time polymerase chain reaction (PCR) analysis}

The total RNA of cells was extracted using TRIzol ${ }^{\text {TM }}$ reagent (Life Technologies, Gaithersburg, MD) as per the manufacturer's instructions. RNA ( $1 \mu \mathrm{g})$ isolated from each sample was reversetranscribed using oligo $(\mathrm{dT})_{15}$ primers with AccuPower ${ }^{\circledR}$ RT PreMix (iNtRON Biotechnology, Gyeonggi-do, South Korea). Next, the generated cDNAs were amplified with AccuPower ${ }^{\circledR}$ PCR PreMix (Bioneer Corporation, Daejeon, South Korea). For mRNA quantification, total RNA was extracted using the RNAqueous ${ }^{\circledR}$ kit, and cDNA was synthesized using $1 \mu \mathrm{g}$ of total RNA with the high capacity RNA-to-cDNA kit (Applied Biosystems, Foster City, CA) according to the manufacturer's protocol. Quantitative real-time PCR was performed using a LightCycler ${ }^{\circledR}$ 1.5 System (Roche Diagnostics GmbH, Mannheim, Germany). Thermocycling conditions consisted of an initial denaturation of $10 \mathrm{~s}$ at $95{ }^{\circ} \mathrm{C}$, followed by 45 cycles of $95{ }^{\circ} \mathrm{C}$ for $10 \mathrm{~s}, 60^{\circ} \mathrm{C}$ for $5 \mathrm{~s}$ and $72{ }^{\circ} \mathrm{C}$ for $10 \mathrm{~s}$. For the calculation of relative quantification, the $2^{-\Delta \Delta C T}$ formula was used, where ${ }^{-} \Delta \Delta C T=\left(C_{\mathrm{T}, \text { target }}-C_{\mathrm{T}, \beta \text {-actin }}\right)$ experimental sample $-\left(C_{\mathrm{T} \text {,target }}\right.$ $\left.-C_{\mathrm{T}, \beta \text {-actin }}\right)$ control sample.

\section{Statistical analysis}

The data were analyzed using GraphPad Prism version 5 software (GraphPad Software, Inc., San Diego, CA). Data have been presented as the mean \pm S.E.M. Statistical significance was evaluated using one-way analysis of variance (ANOVA) and the differences were assessed by Dunnett's test. A value of $P<0.05$ was considered to indicate statistical significance.

\section{Results}

THIA does not affect cytotoxicity in BMMs and RANKLinduced osteoclastogenesis

To examine the potential effects of THIA (Fig. 1A) in osteoclast differentiation, the cell toxicity of THIA was determined in primary mouse bone marrow macrophages (BMMs) derived from mouse whole bone marrow cells. MTT assay showed no cytotoxic effects up to a concentration of $30 \mu \mathrm{M}$ in BMMs (Fig. 1B) as well as under osteoclast differentiation for 3 and 5 days on using $100 \mathrm{ng} \mathrm{mL}^{-1}$ RANKL (Fig. 1C and D).

\section{THIA inhibits RANKL-induced osteoclast gene expression and TRAP (+) MNCS}

To examine the effects of THIA on mRNA expression for osteoclast differentiation, BMMs were differentiated for 3 days using $100 \mathrm{ng} \mathrm{mL}{ }^{-1}$ RANKL in the presence or absence of $10 \mu \mathrm{M}$ and $30 \mu \mathrm{M}$ THIA. As shown in Fig. 2A and B, THIA downregulated gene expression of c-Fos and NF-ATc1 (the initiation factor of osteoclast differentiation). On differentiation for 5 days by $100 \mathrm{ng} \mathrm{mL}^{-1}$ RANKL, osteoclastogenesis was analyzed using TRAP assays. The results have shown that THIA suppressed the TRAP-positive staining (Fig. 2C) and the proportion of TRAPpositive multinucleated osteoclasts (MNCs) was compared to the number of nuclei (Fig. 2D).

\section{THIA inhibits RANKL-induced F-actin ring formation}

F-Actin ring formation assay was carried out during RANKLinduced osteoclastogenesis since the change in the cytoskeletal structure was essential for bone resorptive functions of the osteoclasts. To investigate functional osteoclast activities, osteoclasts were stained with FITC-phalloidin and DAPI. Immunofluorescence analysis via microscopy analyses revealed that THIA significantly attenuated the F-actin ring formation by RANKL (Fig. 3A and B). These results have suggested that THIA suppressed RANKLinduced osteoclastogenesis in primary cultured mouse BMMs.

\section{THIA has no cytotoxicity in primary mouse calvarial pre-} osteoblasts

To assess the potential effects of THIA in osteoblast differentiation, cell viability was examined in primary mouse calvarial pre-osteoblasts. MTT assays have shown that no cytotoxic effects were observed in the calvarial pre-osteoblasts (Fig. 4A) as well as under osteoblast differentiation for 5 days in osteogenic supplement medium (OS) containing $50 \mu \mathrm{g} \mathrm{mL} \mathrm{m}^{-1}$ L-ascorbic acid (L-AA) and $10 \mathrm{mM} \beta$-glycerophosphate ( $\beta$-GP) (Fig. 4B).

THIA does not affect osteoblast differentiation in the calvarial pre-osteoblasts

Next, we investigated the effects of THIA on osteoblast differentiation in the presence and absence of THIA. The effect of THIA on osteoblast differentiation was detected by measuring the ALP enzymatic activity as an early osteoblast differentiation marker. As shown in Fig. 5A, THIA did not affect the ALP activity. Next, the degree of mineralized nodule formation was determined for 14 
A

B
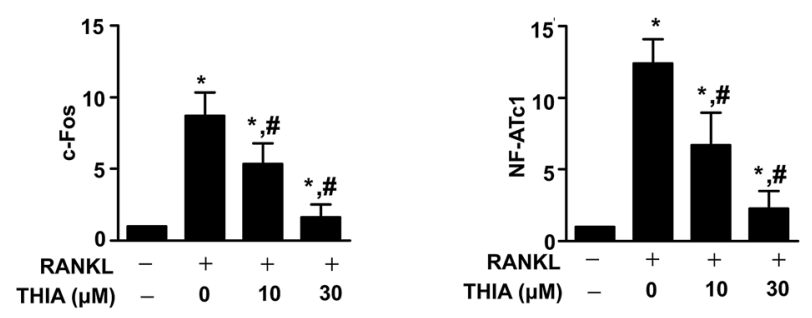

C

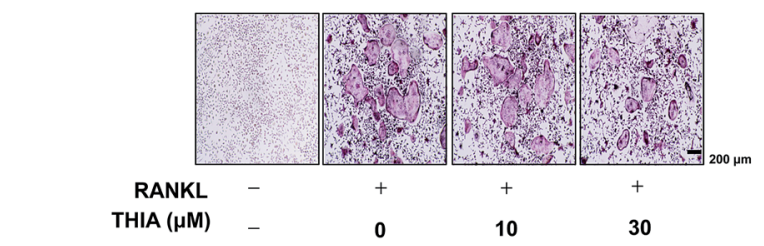

D
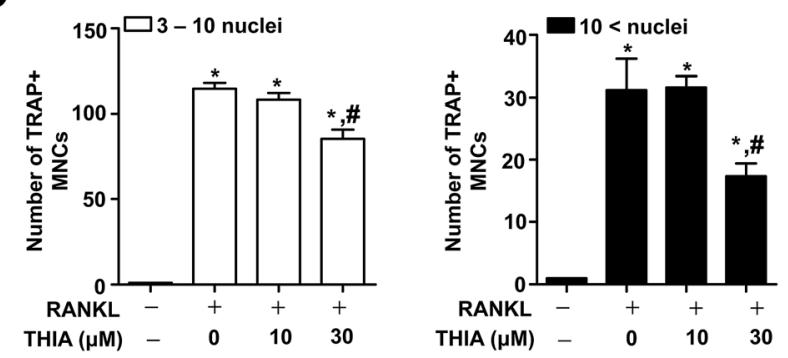

Fig. 2 Effects of THIA on RANKL-induced osteoclast gene expression and TRAP positive MNCs. (A and B) BMMs were seeded onto 6 well plates and cultured in $30 \mathrm{ng} \mathrm{mL}^{-1} \mathrm{M}-\mathrm{CSF}$ and $100 \mathrm{ng} \mathrm{mL}^{-1}$ RANKL with 10 and $30 \mu M$ THIA for 3 days. Total RNA was isolated and c-Fos (A) and NF-ATC1 (B) were analyzed by qRT-PCR. The values obtained for the target gene expression were normalized to $\beta$-actin and relatively quantified to the expression in non-stimulated control group. ( $C$ and D) The BMMs were seeded onto 48 well plates and cultured in $30 \mathrm{ng}$ $\mathrm{mL}^{-1} \mathrm{M}-\mathrm{CSF}$ and $100 \mathrm{ng} \mathrm{mL}^{-1}$ RANKL with 10 and $30 \mu \mathrm{M}$ THIA for 5 days. Mature osteoclasts were detected with TRAP staining (A). TRAP positive MNCs and the number of MNCs are shown. 0-10 nuclei (left), $10<$ nuclei (right) (B). Scale bar: $200 \mu \mathrm{m}$. MNCs: multinucleated cells. *, $p<0.05$ compared to control. \#, $p<0.05$ compared to RANKL. Data are representative of three independent experiments.

days by measuring the ARS staining as a late osteoblast marker. Consistent with the effects on ALP activity, THIA had no influence on the mineralized nodule formation (Fig. 5B). The quantification of mineralized nodule formation statistically validated the effect of THIA on osteoblast differentiation (Fig. 5C), suggesting that THIA has no effects on osteoblasts.

\section{Discussion}

Natural compounds, in particular organosulfur compounds, such as alliin, $\gamma$-glutamyl- $S$-allylcysteine, $S$-methylcysteine sulfoxide, and $S$-allylcysteine, isolated from garlic, can be considered as potential pharmaceutical drug candidates to prevent and treat various diseases. ${ }^{7}$ Our group has investigated an organosulfur compound, thiacremonone (THIA), isolated from
A
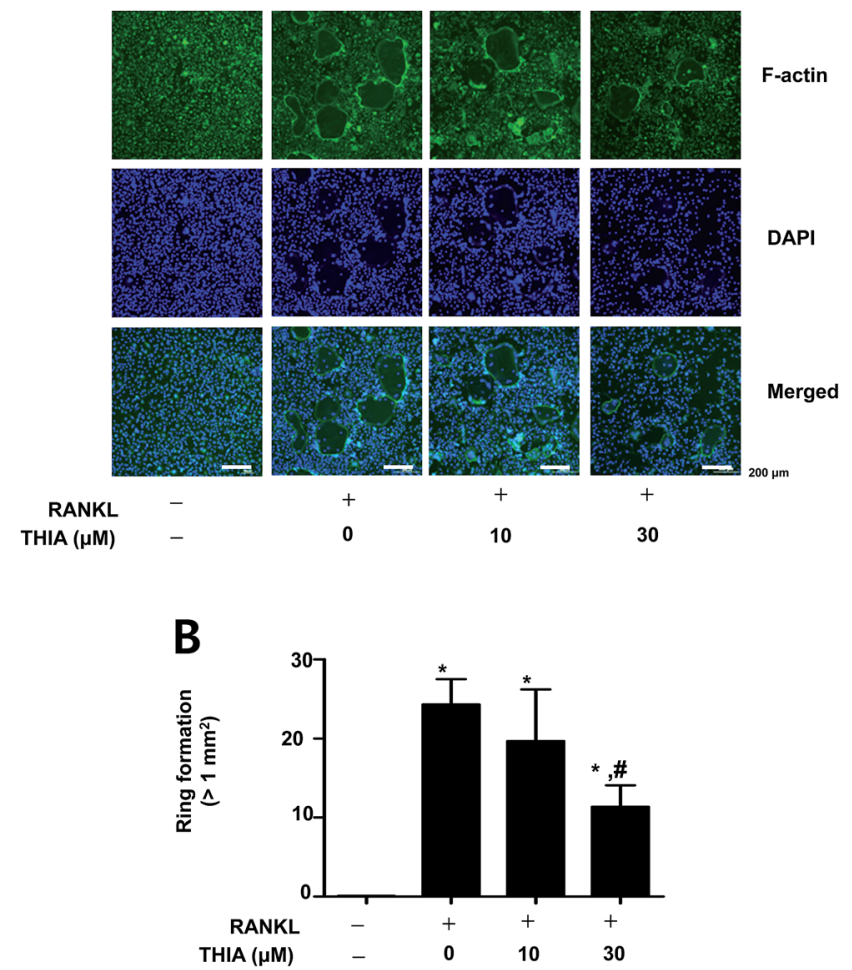

Fig. 3 Effects of THIA on RANKL-induced F-actin ring formation. (A and B) F-Actin ring formation (green) was observed by a fluorescence microscope after staining with FITC-phalloidin (green) and DAPI (blue) $(A)$, and then number of ring formation $\left(>1 \mathrm{~mm}^{2}\right)$ was measured $(B)$. Scale bar: $100 \mu \mathrm{m} .{ }^{*}, p<0.05$ compared to control. \#, $p<0.05$ compared to RANKL. Data are representative of three independent experiments.

heated garlic that demonstrated to be promising candidates for drugs in the therapy of cancer, arthritic diseases, neurological diseases and liver diseases. ${ }^{\mathbf{8 - 1 1}}$ Earlier, we have reported that 2,4,5-trimethoxyldalbergiquinol isolated from Dalbergia odorifera was used as a traditional herbal medicine aiding osteoblast differentiation and mineralized nodule formation. ${ }^{16}$ In the present study, we have investigated the potential effects of THIA in primary mouse calvarial pre-osteoblasts and bone marrow macrophages (BMMs) for osteoblast and osteoclast differentiation since calvarial pre-osteoblasts and BMMs isolated from calvaria and bone marrow were widely used for osteoblast and osteoclast differentiation for in vitro assay system. ${ }^{13,15}$ In the present study, we have demonstrated the inhibitory effect of THIA on RANKL-induced osteoclastogenesis in the BMMs, regardless of osteoblast differentiation in the calvarial preosteoblasts.

Osteoclasts develop from monocyte-lineage hematopoietic precursors during a multistep differentiation process called osteoclastogenesis. ${ }^{17}$ RANKL binds to its receptor RANK on immature osteoclast and precursor cells and induces transcription factors such as c-Fos and NFATc1. ${ }^{18,19} \mathrm{c}-\mathrm{Fos}$, which is induced by RANKL, plays an essential role in the initiation of osteoclast differentiation and is responsible for the expression of another critical regulator. ${ }^{20-22}$ In c-Fos knockout mouse, 


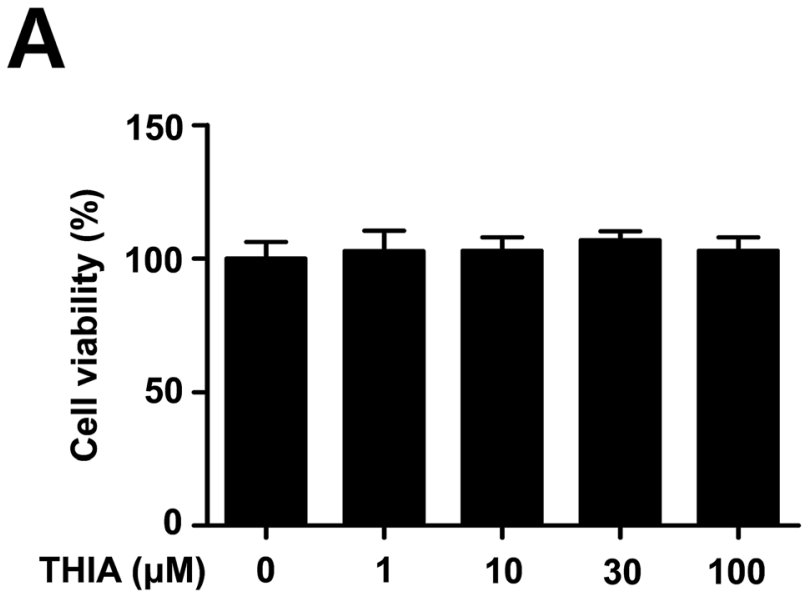

D

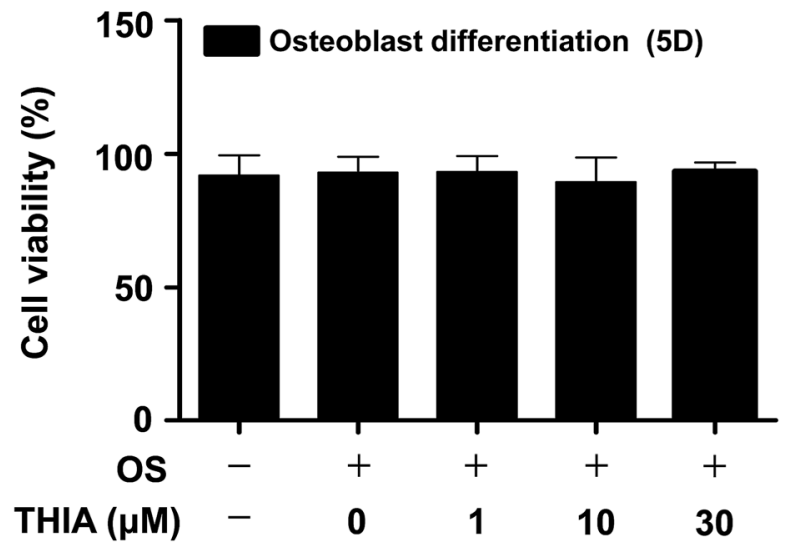

Fig. 4 Effects of THIA on cytotoxicity in calvarial pre-osteoblasts and osteoblasts. (A) After primary mouse calvarial pre-osteoblasts were seeded onto 96-well plates, and THIA was treated in the cells with the indicated doses for $24 \mathrm{~h}$. (B) The cells were cultured with THIA in osteogenic supplement medium (OS) containing $50 \mu \mathrm{g} \mathrm{mL} \mathrm{m}^{-1} \mathrm{~L}$ ascorbic acid (L-AA) and $10 \mathrm{mM} \beta$-glycerophosphate ( $\beta$-GP) for 5 days. Cell viability was measured using the MTT assay. Data are representative of three independent experiments.

NFATc1 is down-regulated, leading to an impaired osteoclast differentiation and function. ${ }^{23}$ The results have shown that THIA markedly attenuates RANK-induced c-Fos and NF-ATc1 expressions. These data suggest that the RANKL-induced osteoclastogenesis is attenuated by THIA through the downregulation of c-Fos and NF-ATc1.

Osteoclasts are large multinucleated cells (MNCs) that resorb mineralized bone and have important roles in bone remodeling and skeletal morphogenesis. ${ }^{17}$ In the present study, we have found that THIA suppressed TRAP-positive MNCs formation as well as F-actin ring formation by RANKL in BMMs. Consistently, RANKL-induced mature osteoclasts generated TRAP-positive MNCs and reorganized the actin cytoskeleton to attach to the bone surface and then resorbed the mineralized bone. ${ }^{24,25}$ Therefore, our results suggest that THIA suppresses RANKLinduced osteoclast differentiation and function via the inhibition of large multinucleated cell formation.
A

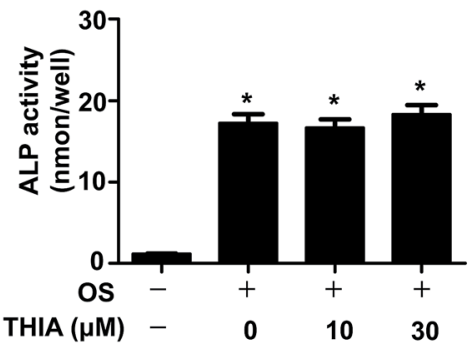

B

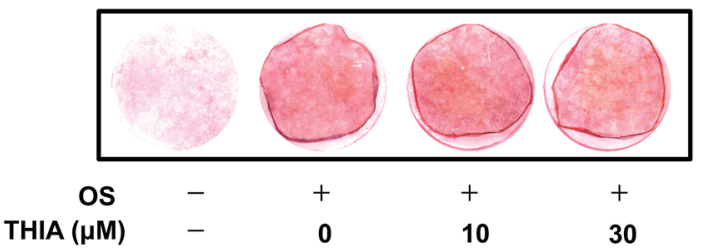

C

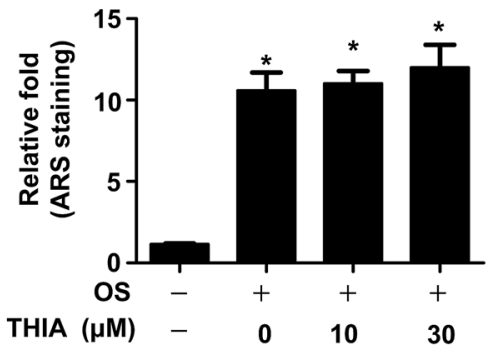

Fig. 5 Effects of THIA on osteoblast differentiation in primary mouse calvarial pre-osteoblasts. (A) The cells were seeded onto 48 well plates and cultured in OS containing $50 \mu \mathrm{g} \mathrm{mL}^{-1}$ L-AA and $10 \mathrm{mM} \beta$-GP with THIA for 7 days ALP activity was measured via ALP activity assays kit. (B and C) After the cells were seeded onto 12 well plates, the cells were cultured in OS with 0,10 , and $30 \mu \mathrm{M}$ THIA for 14 days. Mineralized nodule formation was assessed by Alizarin red S (ARS) staining (B), and stains were eluted with DMSO to quantify the amount of alizarin red staining and measured at $590 \mathrm{~nm}$. The data are represented as relative fold of the control (C). $*, p<0.05$ compared to control. Data are representative of three independent experiments.

Osteoblast differentiation and the mineralized extracellular matrix formation are also involved in the bone formation. ALP is the early stage marker for osteoblast differentiation, and the mineralized nodule formation is the late stage marker for mature osteoblast. ${ }^{26,27}$ The results have shown that THIA did not influence the ALP activity and mineralized nodule formation in the calvarial pre-osteoblasts, suggesting that THIA does not have pharmacological properties in osteoblast differentiation.

In conclusion, recent studies have indicated that functionally active components isolated from garlic have beneficial effects in the treatment of various diseases, thereby having a wide range of applications. $^{7}$ The present study is the first report for selective inhibition of RANKL-induced osteoclastogenesis by THIA via the downregulation of c-Fos and NF-ATc1 in primary cultures of BMMs, but not in mouse calvarial pre-osteoblasts. A schematic 


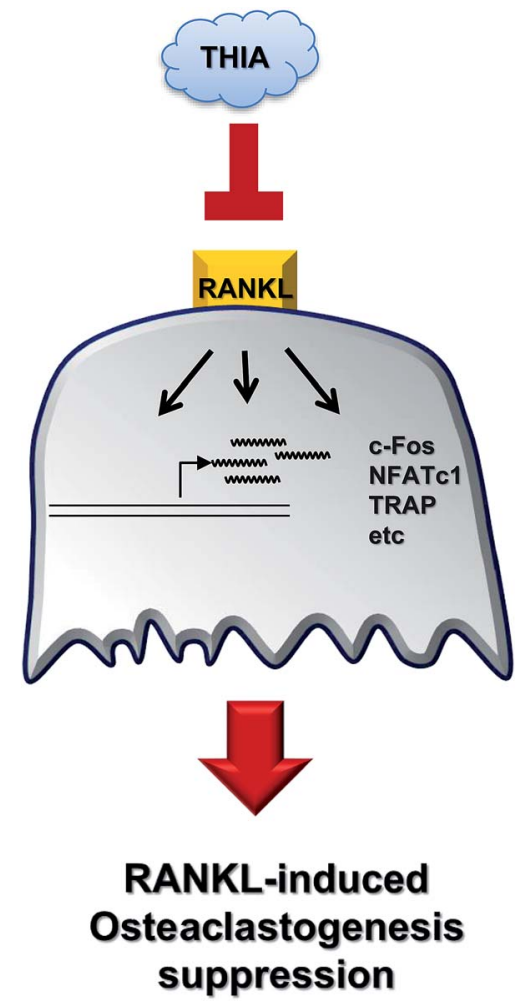

Fig. 6 Schematic for RANKL-induced osteoclastogenesis modulation by THIA.

showing the effects of THIA on RANKL-induced osteoclastogenesis is shown in Fig. 6. Therefore, these findings suggest that THIA might be useful as an effective agent in the treatment of bone diseases such as osteoporosis, periodontal disease, and osteoarthritis. Furthermore, our data will be a foundation for further studies to determine roles underlying the effects and clinical applications of functionally active components isolated from garlic.

\section{Conflict of interest}

The authors declare no competing financial interests.

\section{Acknowledgements}

This study was supported by the National Research Foundation of Korea (NRF) grant funded by the Korea government (MEST) (MRC, 20080062275; 2015R1D1A1A01059240).

\section{References}

1 P. J. Marie and M. Kassem, Eur. J. Endocrinol., 2011, 165, 1-10. 2 S. F. Vondracek, P. Minne and M. T. McDermott, Clin. Interventions Aging, 2008, 3, 315-329.

3 S. Khosla and B. L. Riggs, Endocrinol. Metab. Clin. North Am., 2005, 34, 1015-1030.

4 B. L. Riggs and A. M. Parfitt, J. Bone Miner. Res., 2005, 20, 177-184.
5 B. L. Riggs and L. C. Hartmann, N. Engl. J. Med., 2003, 348, 618-629.

6 M. Augustine and M. J. Horwitz, Curr. Osteoporos Rep., 2013, 11, 400-406.

7 H. M. Yun, J. O. Ban, K. R. Park, C. K. Lee, H. S. Jeong, S. B. Han and J. T. Hong, Pharmacol. Ther., 2014, 142, 183-195.

8 J. O. Ban, J. H. Oh, T. M. Kim, D. J. Kim, H. S. Jeong, S. B. Han and J. T. Hong, Arthritis Res. Ther., 2009, 11, R145.

9 M. Jo, H. M. Yun, K. R. Park, M. H. Park, D. H. Lee, S. H. Cho, H. S. Yoo, Y. M. Lee, H. S. Jeong, Y. Kim, J. K. Jung, B. Y. Hwang, M. K. Lee, N. D. Kim, S. B. Han and J. T. Hong, PLoS One, 2014, 9, e91508.

10 Y. R. Kim, N. J. Lee, J. O. Ban, H. S. Yoo, Y. M. Lee, Y. P. Yoon, S. Y. Eum, H. S. Jeong, D. Y. Yoon, S. B. Han and J. T. Hong, J. Evidence-Based Complementary Altern. Med., 2013, 2013, 974794.

11 H. M. Yun, P. Jin, K. R. Park, J. Hwang, H. S. Jeong, E. C. Kim, J. K. Jung, K. W. Oh, B. Y. Hwang, S. B. Han and J. T. Hong, Mol. Neurobiol., 2016, 53, 2409-2420.

12 G. H. Lin, Y. J. Lee, D. Y. Choi, S. B. Han, J. K. Jung, B. Y. Hwang, D. C. Moon, Y. Kim, M. K. Lee, K. W. Oh, H. S. Jeong, J. Y. Leem, H. K. Shin, J. H. Lee and J. T. Hong, J. Alzheimer's Dis., 2012, 29, 659-676.

13 H. M. Yun, K. R. Park, J. T. Hong and E. C. Kim, Sci. Rep., 2016, 6, 30985.

14 H. M. Yun, K. R. Park, T. H. Quang, H. Oh, J. T. Hong, Y. C. Kim and E. C. Kim, Arch. Pharmacal Res., 2017, 40, 601-609.

15 E. J. Chang, J. Ha, F. Oerlemans, Y. J. Lee, S. W. Lee, J. Ryu, H. J. Kim, Y. Lee, H. M. Kim, J. Y. Choi, J. Y. Kim, C. S. Shin, Y. K. Pak, S. Tanaka, B. Wieringa, Z. H. Lee and H. H. Kim, Nat. Med., 2008, 14, 966-972.

16 H. M. Yun, K. R. Park, T. H. Quang, H. Oh, J. T. Hong, Y. C. Kim and E. C. Kim, Cell Death Dis., 2015, 6, e1819.

17 S. L. Teitelbaum and F. P. Ross, Nat. Rev. Genet., 2003, 4, 638-649.

18 A. J. Whitmarsh and R. J. Davis, J. Mol. Med., 1996, 74, 589-607. 19 P. Monje, J. Hernandez-Losa, R. J. Lyons, M. D. Castellone and J. S. Gutkind, J. Biol. Chem., 2005, 280, 35081-35084.

20 H. Takayanagi, Nat. Rev. Rheumatol., 2009, 5, 667-676.

21 H. Takayanagi, S. Kim, T. Koga, H. Nishina, M. Isshiki, H. Yoshida, A. Saiura, M. Isobe, T. Yokochi, J. Inoue, E. F. Wagner, T. W. Mak, T. Kodama and T. Taniguchi, Dev. Cell, 2002, 3, 889-901.

22 H. B. Kwak, B. K. Lee, J. Oh, J. T. Yeon, S. W. Choi, H. J. Cho, M. S. Lee, J. J. Kim, J. M. Bae, S. H. Kim and H. S. Kim, Bone, 2010, 46, 724-731.

23 K. Matsuo, D. L. Galson, C. Zhao, L. Peng, C. Laplace, K. Z. Wang, M. A. Bachler, H. Amano, H. Aburatani, H. Ishikawa and E. F. Wagner, J. Biol. Chem., 2004, 279, 26475-26480.

24 W. J. Boyle, W. S. Simonet and D. L. Lacey, Nature, 2003, 423, 337-342.

25 S. L. Teitelbaum, Science, 2000, 289, 1504-1508.

26 H. S. Lee, E. Y. Jung, S. H. Bae, K. H. Kwon, J. M. Kim and H. J. Suh, Phytother. Res., 2011, 25, 716-723.

27 M. B. Kim, Y. Song and J. K. Hwang, Fitoterapia, 2014, 98, 5965. 